INPLASY

PROTOCOL

To cite: Li et al. Acupuncture and moxibustion for Primary Osteoporosis: A systematic review and meta-analysis. Inplasy protocol 202210018. doi:

10.37766/inplasy2022.1.0018

Received: 05 January 2022

Published: 05 January 2022

Corresponding author:

Wei Li

fengxieqing10yang@163.com

Author Affiliation:

Henan Vocational College of Nursing.

Support: Anyang science and Technology.

Review Stage at time of this submission: The review has not yet started.

Conflicts of interest:

None declared.

\section{Acupuncture and moxibustion for Primary Osteoporosis: A systematic review and meta-analysis}

$\mathrm{Li}, \mathrm{X}^{1}$; Li, XY2; Chen, $\mathrm{XL}^{3}$; Xing, AJ4; Dong, $\mathrm{HH}^{5}$; Wang, $\mathrm{YL}^{6}$; Ren, JK7 Wang, YK' ${ }^{8}$; Zhang, $Q^{9}$.

Review question / Objective: Is acupuncture effectiveness and safty for osteoporosis?

Condition being studied: Primary osteoporosis (PO) is a common disease that was characterized by a systemic impairment of bone mass and microarchitecture that results in fragility fractures and constitutes a pressing public health problem. But the effect of acupuncture or moxibustion treatment for PO is controversial.

Information sources: Five electronic international (Web of Science, The Cochrane Library, PubMed, MEDLINE, and EMBASE) will be searched from their inception until Jan. 2022.

INPLASY registration number: This protocol was registered with the International Platform of Registered Systematic Review and Meta-Analysis Protocols (INPLASY) on 05 January 2022 and was last updated on 05 January 2022 (registration number INPLASY202210018).

\section{INTRODUCTION}

Review question / Objective: Is acupuncture effectiveness and safty for osteoporosis?

Condition being studied: Primary osteoporosis (PO) is a common disease that was characterized by a systemic impairment of bone mass and microarchitecture that results in fragility fractures and constitutes a pressing public health problem. But the effect of acupuncture or moxibustion treatment for $\mathrm{PO}$ is controversial. 


\section{METHODS}

Participant or population: Participants who are adults diagnosed with $\mathrm{PO}$ using definitive diagnostic criteria or radiographically report the Chinese Medical Association criteria and guiding principles of clinical research on new drugs - PO was included. There is no restriction on gender or race.

Intervention: The studies of Acupuncture (electro-acupuncture, auricular acupuncture, warm-acupuncture and dry needling, etc.) and moxibustion used as an intervention to treat the PO were included. We also included trials that compared acupuncture and moxibustion plus another active treatment versus other active treatment alone.

Comparator: As to the control interventions, any form of therapy that didn't involve acupuncture and moxibustion were included like medication, sham acupuncture, usual care, or no intervention.

Study designs to be included: We included all RCTs with eligible intervention(s) and outcome(s) for PO published in Chinese and English. For the crossover trials with the randomized control design, the first stage consisted of data collection for this meta-analysis. Furthermore, we only included RCTs with a Jadad score $\geq 4$ since the previously published systematic review could not obtain accurate conclusions due to the low quality of RCTs included.

\section{Eligibility criteria: According to the PICO.}

Information sources: Five electronic international (Web of Science, The Cochrane Library, PubMed, MEDLINE, and EMBASE) will be searched from their inception until Jan. 2022.

Main outcome(s): BMD.

Additional outcome(s): Clinical therapeutic effect, level of serum calcium, treatment effectiveness, pain score, level of serum alkaline phosphatase, osteocalcin. We compiled a table of any excluded studies, about which was plausible to expect that a reader would question why the study was not included.

Quality assessment / Risk of bias analysis: Two reviewers will evaluate the included study according to the bias risk assessment tool recommended by Cochrane manual independently. A judgement of low-risk, high-risk and unclear-risk by assigning for each result. It consists of the following six aspects: 1 . Selection bias: random sequence generation and allocation concealment; 2 . Performance bias: the blind method which is applied to the participants and treatment providers. 3. Detection bias: blinding of outcome assessment. No need to do blind method and blind method is effective is low -risk, while blind method is ineffective is high-risk. 4. Attrition bias: incomplete outcome data. If less than $5 \%$ of the participants lost and the causes of the two treatment groups were similar, the risk is low. It is a high risk of bias if more than $10 \%$ participants lost, or the reasons for losing follow-up are different between treatment groups. If the authors did not report a loss of follow-up or the number of follow-up losses is between $5 \%$ and $10 \%$, the risk is not clear. 5. Reporting bias: whether results are selectively reported. 6 . Other biases. Other information related to interests, such as fund sources, magazine interest points.

Strategy of data synthesis: We will make a statistical analysis for the data with STATA 15.0. The data synthesis which shows the comprehensive effect for the including studies will be demonstrated by a forest plot. We will use RR and $95 \% \mathrm{Cl}$ as a pooled effect estimate for dichotomous outcomes, while $M D$ and $95 \% \mathrm{Cl}$ for continuous outcomes. If the studies have no substantial heterogeneity $\left(I^{2}<50 \%\right)$, we will use fixed-effect model. Instead, we will use random-effect model. Descriptive analysis shall be implemented when meta-analysis is not appropriate. Subgroup analysis of different interventions, such as acupuncture and acupressure, and subgroup analysis of different controls, 
such as drug therapy, sham acupuncture control, routine care, etc.

Subgroup analysis: Subgroup analysis of different interventions, such as acupuncture and acupressure, and subgroup analysis of different controls, such as drug therapy, sham acupuncture control, routine care, etc.

Sensitivity analysis: None.

Country(ies) involved: China.

Keywords: Acupuncture, Osteoporosis, meta-analysis.

Contributions of each author:

Author 1 - Wei Li.

Author 2 - Xiaoya Li.

Author 3 - Xinli Chen.

Author 4 - Aojing Xing.

Author 5 - Haonan Dong.

Author 6 - Yalan Wang.

Author 7 - Jiankun Ren.

Author 8 - Yanke Wang.

Author 9 - Qi Zhang. 